\title{
Green Technologies for Wastewater Depollution `
}

\author{
Radu Claudiu Fierascu 1,2, Roxana Ioana Brazdis 1,2,*, Irina Fierascu 1,3,*, Anda Maria Baroi 1,3, \\ Toma Fistos ${ }^{1,2}$ and Sorin Marius Avramescu ${ }^{4}$ \\ 1 National Institute for Research \& Development in Chemistry and Petrochemistry - ICECHIM, \\ 202 Spl. Independentei, 060021 Bucharest, Romania; fierascu.radu@icechim.ro (R.C.F.); \\ anda.baroi@icechim.ro (A.M.B.); toma.fistos@icechim.ro (T.F.) \\ 2 University "Politehnica" of Bucharest, Spl. Independentei 313, 011061 Bucharest, Romania \\ 3 University of Agronomic Science and Veterinary Medicine, 59 Marasti Blvd., 011464 Bucharest, Romania \\ 4 Department of Organic Chemistry, Biochemistry and Catalysis, Faculty of Chemistry, University of \\ Bucharest, 90-92 Panduri Street, 50663 Bucharest, Romania; sorin_avramescu@yahoo.com \\ * Correspondence: roxana.brazdis@icechim.ro (R.I.B.); dumitriu.irina@yahoo.com (I.F.) \\ + Presented at the 16th International Symposium "Priorities of Chemistry for a Sustainable Development" \\ PRIOCHEM, Bucharest, Romania, 28-30 October 2020.
}

Published: 10 November 2020

Keywords: environment; wastewater depollution; green technologies

Water pollution remains nowadays a critical issue of our society, since in the last decades the pace of industrialization, urbanization and agricultural activities increased rapidly. Consequently, water shortages and water pollution are currently the two major water-related problems experienced by numerous countries worldwide. Long-term adverse effects of xenobiotics in aquatic ecosystems imply that current water treatment methods have to be improved in order to remove these pollutants at their source or in municipal water treatment plants.

Using a new water treatment technology for the removal of NSAIDs (nonsteroidal antiinflammatory drugs) based on a catalytic ozonation process with catalytic systems arranged in the form of thin films deposited on a glass substrate is a promising "green technology". Thin-film morphology with phytosynthesized, nanosized, mixed oxide particles are suitable for catalyst processes since this approach includes advantages from superior properties of nanoparticles and avoidance of diffusivity problems (in fixed bed systems) or the daunting separation step (in slurry approach). Another important innovative and ecofriendly aspect of this technology is the preparation of novel thin-film ozonation catalysts using plant extracts as an environment for nanoparticle formation and stabilization from catalytic starter materials. After preparation, nanoparticles can be used as such (in some cases), but the unwanted possibility to agglomerate or to be deposited in porous structures or on thin films remain. In porous structures, nanoparticles are formed inside pores as a result of a multistep, costly and difficult process without having very good control over their chemical composition, dimensions and shape. Moreover, after preparation and integration in a chemical process, their performances are seriously reduced by diffusion processes, pore blocking and deactivation.

This type of arrangement brings incontestable benefits compared with porous supports: (a) The deposition of nanoparticles can be accomplished by several methods (spin coating, dip coating, etc.) that are easy to apply and use established technology; (b) composition, thickness, functionalization and morphology of thin films can be easily modulated in order to obtain desired properties; (c) the range of precursors for thin-film production is virtually limitless and therefore the number of applications is staggering; (d) thin films can be implemented in all kinds of experimental set-ups and, in the case of chemical reactors, restraints like diffusion phenomena, hydrodynamic behavior of fluid flow inside packed bed and pressure drop associated with fixed bed arrangements do not occur; (e) 
regeneration of solids and reactor maintenance (if needed) during the life cycle and recycling after total deactivation are easier in case of the thin-film approach which makes them a greener choice compared with porous supports; (f) characterization of thin films with different techniques is easier compared with nanoparticles embedded in porous structures. These features make thin films suitable to be used in water treatment processes, especially in the oxidation of pollutants, and the design of most photocatalytic processes benefits already from the presence of these features.

Acknowledgments: This work was supported by a grant of the Romanian National Authority for Scientific Research and Innovation, CNCS/CCCDI-UEFISCDI, project number PN-III-P2-2.1-PED-2019-3166, contract 299 PED/2020, within PNCDI III.

Publisher's Note: MDPI stays neutral with regard to jurisdictional claims in published maps and institutional affiliations.

(C) 2020 by the authors. Licensee MDPI, Basel, Switzerland. This article is an open access article distributed under the terms and conditions of the Creative Commons Attribution (CC BY) license (http://creativecommons.org/licenses/by/4.0/). 